\title{
The Role of Optical Coherence Tomography Angiography to Assess Vessel Density and Retinal Perfusion in Diabetic Retinopathy
}

\author{
Emiliano Di Carlo ${ }^{1,2 *}$ and Augustin $\mathrm{AJ}^{1}$ \\ ${ }^{1}$ Department of Ophthalmology, Germany \\ ${ }^{2}$ Ophthalmology Unit, Department of Experimental Medicine and Surgery, University of Rome Tor Vergata, Italy
}

Submission: July 16, 2018; Published: July 23, 2018

*Corresponding author: Emiliano Di Carlo, Department of Ophthalmology, Staedtisches Klinikum Karlsruhe, Moltkestraße 90, 76133 Karlsruhe, Germany, Tel: 00497219742010; Fax: 00497219742019; Email: emi.dicarlo@hotmail.it

\begin{abstract}
Diabetic retinopathy (DR) is a chronic microangiopathy that progressively leads to increased vascular permeability, retinal ischemia and, in advanced cases, to retinal neovascularization. Optical coherence tomography angiography (OCTA) is a novel angiography technique based on the principles of OCT that has the ability to enhance the diabetic eyes knowledge by providing high-resolution images of retinal microvasculature blood flow and morphology. In addition to its velocity, feasibility and safety compared to conventional fluorescein angiography (FA), OCTA particularly provides better visualization of superficial and deep retinal layers and offers the possibility to quantitatively measure the areas of non perfusion at the level of the macula and posterior pole. Moreover, the presence of softwares able to quantify the retinal capillary perfusion, such as the capillary perfusion density maps and average perfusion density values, allows to detect all the minimal vascular changes. The role of OCTA to detect retinal vascular changes in DR has been exhaustively demonstrated, showing the ability to accurately assess the impaired capillary perfusion, intraretinal vascular anomalies, neovascularization, some types of microaneurysms and features of macular edema related to the retinal ischemia. The aim of this review is to summarize the main aspects regarding the role of OCTA to detect the typical characteristics of vessel density and retinal perfusion in DR.
\end{abstract}

Keywords: Diabetic retinopathy; Optical coherence tomography angiography; Retinal perfusion; Vessel density; Foveal avascular zone; Nonperfusion areas; Retinal periphery

Abbreviations: DR: Diabetic Retinopathy; OCTA: Optical Coherence Tomography Angiography; FA: Fluorescein Angiography; NPAs: NonPerfusion Areas; NV: Neovascularization; SVP: Superficial Vascular Plexus; ICP: Intermediate Capillary Plexus; DCP: Deep Capillary Plexus; FAZ: Foveal Avascular Zone; SS-OCTA: Swept-Source Optical Coherence Tomography Angiography; DME: Diabetic Macular Edema; DMI: Diabetic Macular Ischemia; PI: Perfusion Index; EFI: Extended Field Imaging

\section{Introduction}

DR represents one of the leading causes of visual impairment and blindness in the world. Circa 285 million people worldwide result to be affected by diabetes mellitus. Approximately $33 \%$ have signs of any DR and $11 \%$ have vision-threatening DR [1]. DR is essentially a microangiopathy that causes capillary occlusion, vascular hyper permeability, and neovascularization in the retina. It is crucial to detect non-perfusion areas (NPAs) or retinal neovascularization $(\mathrm{NV})$ in order to evaluate $\mathrm{DR}$ progression and decide on courses of treatment [2]. Although FA remains currently the gold standard imaging technique in the evaluation, management and follow-up of patients affected by retinal vascular diseases, such as diabetic retinopathy, is

an invasive examination, unrepeatable on the same day or in the short term, and can cause several complications. OCTA is a new imaging technique based on OCT which allows for the visualization of functional blood vessels, without the need to use a fluorescein dye. The principle of OCTA is to utilize the variation in OCT signal caused by moving particles, such as red blood cells, as the contrast mechanism for imaging blood flow. Specifically, the signal originating from the structural tissue remains steady, while the signal from the blood vessels changes over time due to the constant motion of the RBCs. In order to discriminate the moving particles from static tissue, repeated scans are performed at the same location. 


\section{Current Research in Diabetes \& Obesity Journal}

Temporal changes of the OCT signal in consecutive scans caused by the moving particles produce the angiographic contrast, providing the opportunity to visualize the microvasculature [3]. The retinal vasculature mainly consists of 3 plexuses: the superficial vascular plexus (SVP), located within the ganglion cell layer, the intermediate capillary plexus (ICP) and the deep capillary plexus (DCP), the latter two positioned above and below compared to the inner nuclear layer, respectively. The SVP receives the blood supply from the central retinal artery, while the deeper layers are nourished by vertical anastomoses from the SVP [4]. The ability of the OCTA to obtain a detailed image of the vascular structures of the retina and, at the same time, to provide an exact quantification of both vessel density and perfusion permits to dramatically ameliorate the knowledge of the role played by retinal microvasculature in DR.

\section{Discussion}

One of the most widely recognized uses of the OCTA concerns its capability to accurately assess the shape and size of the foveal avascular zone (FAZ) among diabetic patients with a different stage of disease severity. Bresnick et al. [5] with the aim of FA, demonstrated the enlargement of FAZ in DR and, mostly, showed a significant correlation between the dimensions of the FAZ and the extend of NPAs. Moreover, following studies have confirmed the important role of FAZ enlargement as an indicator of DR progression, leading to the validation of the
FAZ area as a "correlated marker" used to categorize the stage of DR [6]. However, quantitative evaluation of the FAZ based on FA is difficult and rarely done in clinical settings. Takase et al. [7] were the first to report an enlargement of the FAZ area (Figure 1), evaluated by en face OCTA, in diabetic patients with or without DR compared to healthy subjects, evidencing that FAZ alterations have been occurred regardless of the presence of DR and suggesting that diabetic eyes manifest an impairment of the macular microcirculation even before the development of the clinically visible retinopathy. Moreover, De Carlo et al. [8] observed an enlargement and remodeling of the FAZ area also in eyes of subjects with diabetes but without clinical signs of DR. With the aim of a swept-source optical coherence tomography angiography (SS-OCTA), Al Sheikh et al. [9] compared both the area of the FAZ and macular capillary network density at different retinal layers in normal individuals and in DR patients. Confirming the previous reports, the researchers observed a statistically significant enlargement of the FAZ and a decreased vessel density of the capillary network in both the SRL and the DRL in eyes affected by DR. The characteristics of FAZ area in eyes with DR and without diabetic macular edema (DME) have been quantitatively analyzed by the OCTA study of Samara et al. [10], which further evaluated the features of diabetic macular ischemia (DMI) in terms of macular vessel density. They confirmed the previous findings regarding the FAZ enlargement and macular vascular density reduction in eyes affected by DR.

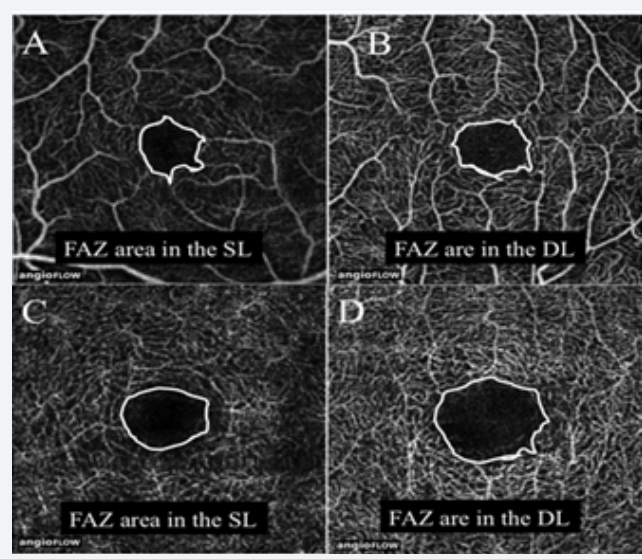

Figure 1: (A-D) representative OCTA images of FAZ morphology.

OCTA images of the FAZ area are represented for each retinal layers (SL=superficial layer and DL=deep layer) in a control subjects (A$B$ ) and in a patients affected by DR (C-D). The two bottom images (C-D) evidence the enlargement of FAZ area, delineated by the white contour, compared to normal subjects (A-D).

Furthermore, both the alterations of the retinal microcirculation, such as FAZ enlargement and vessel density reduction, exhibited a significant correlation with the visual acuity. Falavarjani et al. [11] evaluated the changes in the FAZ area and the retinal capillary density after a single intravitreal anti-VEGF injection for macular edema secondary to DR, finding no significant differences in the retinal vascular perfusion after the intervention, in accordance with previous studies illustrating that anti-VEGF therapy did not worsen capillary non-perfusion.
The assessment of the NPAs represents a fundamental aspect of the DR management and the role of OCTA may offer several advantages compared to conventional FA, because of its ability to detect the features of retinal vascular plexuses. Hwang et al. [12] investigated the feasibility of using OCTA to automatically quantify capillary non-perfusion as a potential sign of DMI, demonstrating a significant reduction of parafoveal and perifoveal vessel densities in DR eyes compared to controls. The study have also showed that OCTA is able to identify NPAs that 


\section{Current Research in Diabetes \& Obesity Journal}

leakage had obscured on FA with a good repeatability, indicating the possibility to objectively analyse the features of the diabetic microvasculopathy (Figure 2).

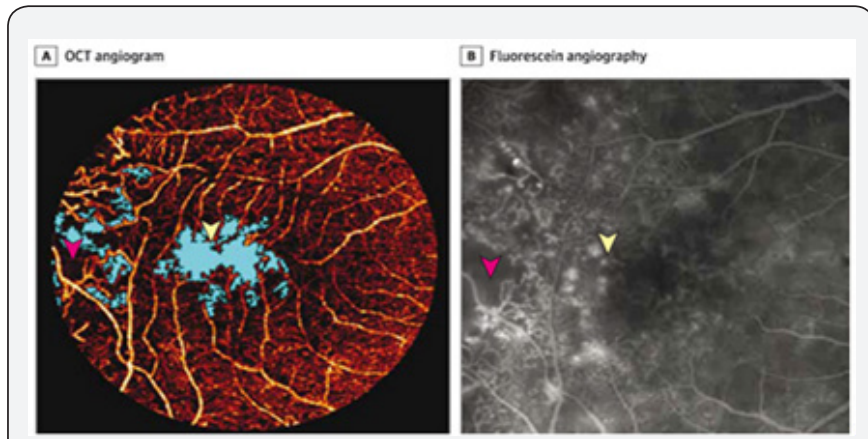

Figure 2: Non perfusion areas (NPAs) seen with both OCTA and FA.

OCTA (A) evidentiating NPAs areas in blue in eye affected by DR. The corresponding FA.

(B). Yellow arrowheads highlight an area of capillary dropout evidenced on OCTA, but not showed by FA. Red arrow heads demonstrate NPAs best seen on FA rather than OCTA.

A recent study by Lin et al. [13] has proposed a specific perfusion index (PI) using the OCT-based microangiography (OMAG) system to evaluate the association between capillary non-perfusion and DR severity. The PI was defined as per cent coverage of area by retinal vessels with flow measured in a $6,8 \times 6,8 \mathrm{~mm} 2$ scan and it has been showed an inverse relationship between increasing DR severity and decreasing capillary perfusion, suggesting that PI may become a useful biomarker in estimating and managing the progression of DR disease. Bhanushali et al. [14] indirectly evaluated capillary NPAs measuring the spacing between the large and small vessels in the different retinal plexuses, asserting that the increased spacing between retinal vessels may be a marker of capillaries loss and decreased capillary perfusion. They found that the spacing between large vessels in the superficial were positively correlated with DR severity. Furthermore, the study illustrated a positive correlation between vessels spacing and diabetes functional parameters, such as fasting blood sugar, postprandial blood sugar and glycosylated hemoglobin, highlighting the role of retinal vessels spacing as a sensitive marker to quantify the progression of DR. The latest developments in the field of OCTA has allowed to obtain a view of the retinal periphery, giving the possibility to study the vascular perfusion also outside the macular area. The study of Sawada et al. [15] compare, for the first time, the ability of a new swept-source wide-field OCTA (12x12mm image scan) to detect NPAs and NV in RD eyes with an ultra-wide field fluorescein angiography system. They demonstrated a high sensitivity (0.98) of wide-field OCTA to detect peripheral vascular alterations, but a not so high specificity (0.82), suggesting at the same time, the clinical useful of wide-field OCTA to evaluate NPAs areas and the need of further studies to better clarify capabilities of the new instrument. Hirano et al. [16] examined the feasibility of wide-field SSOCTA with extended field imaging (EFI) for the evaluation of the retinal vasculature in DR eyes. The EFI technique is a novel yet simple method for extending the scan length of SD-OCT B-scan images involving the imaging of the posterior pole through frial frames fitted with +20 D lenses, able to extend the field of view of wide-field OCTA. The comparison with conventional FA showed values sensitivity and specificity values of SS-OCTA with EFI of $96 \%$ and $100 \%$ respectively. Moreover, the study exhibited no significant differences in the extent of NPAs areas between the two imaging modalities.

\section{Conclusion}

OCTA may be considered a fast, practice and useful tool to analyze the natural course of DR disease. Specifically, it has been showed how a fundamental role could be played by OCTA to detect the distinctive modifications of retinal microvasculature induced by DR. The greatest advancements provided by OCTA are related to the capability to exactly depict the FAZ changes and to quantitatively measure the vessel density and capillary perfusion. Moreover, further steps forward have been already done in the direction to reach the visualization of the retinal periphery by the use of OCTA. The correlation between OCTA parameters and the severity and progression of DR demonstrate the possibility to utilize measurements, such as FAZ area, vessel density and capillary perfusion, in order to ameliorate the management of the disease. Finally, further larger studies are needed to assess the features of peripheral retinal vessels by the use of wide-field OCTA with the aim to better understand the dynamics of retinal microvasculature.

\section{Conflict of Interest}

The authors declare that there is no conflict of interest regarding the publication of this paper.

\section{References}

1. Lee R, Wong TY, Sabanayagam C (2015) Epidemiology of diabetic retinopathy, diabetic macular edema and related vision loss. Eye Vis, Lond 2: 17.

2. Antonetti DA, Klein R, Gardner TW (2012) Diabetic retinopathy. N Engl J Med 366(13): 1227-1239.

3. Kashani AH, Chen CL, Gahm JK, Zheng F, Richter GM, et al. (2017) Optical coherence tomography angiography: A comprehensive review of current methods and clinical applications. Prog Retin Eye Res 60: 66-100.

4. Provis JM (2001) Development of the primate retinal vasculature. Prog Retin Eye Res 20(6): 799-821.

5. Bresnick GH, Condit R, Syrjala S, Palta M, Groo A, et al. (1984) Abnormalities of the foveal avascular zone in diabetic retinopathy. Arch Ophthalmol 102(9): 1286-1293.

6. Mansour AM, Schachat A, Bodiford G, Haymond R (1993) Foveal avascular zone in diabetes mellitus. Retina 13(2): 125-128.

7. Takase N, Nozaki M, Kato A, Ozeki H, Yoshida M, et al. (2015) Enlargement of foveal avascular zone in diabetic eyes evaluated by en face optical coherence tomography angiography. Retina 35(11): 23772383.

8. De Carlo TE, Chin AT, Bonini Filho MA, Adhi M, Branchini L, et al (2015) Detection of microvascular changes in eyes of patients with diabetes but no clinical diabetic retinopathy using optical coherence tomography angiography. Retina 35(11): 2364-2370. 


\section{Current Research in Diabetes \& Obesity Journal}

9. Al Sheikh M, Akil H, Pfau M, Sadda SR (2016) Swept-Source OCT angiography imaging of the foveal avascular zone and macular capillary network density in diabetic retinopathy. Invest Ophthalmol Vis Sci 57(8): 3907-3913.

10. Samara WA, Shahlaee A, Adam MK, Khan MA, Chiang A, et al. (2017) Quantification of diabetic macular ischemia using optical coherence tomography angiography and its relationship with visual acuity. Ophthalmology 124(2): 235-244

11. Ghasemi Falavarjani K, Iafe NA, Hubschman JP, Tsui I, Sadda SR, et al. (2017) Optical coherence tomography angiography analysis of the foveal avascular zone and macular vessel density after AntiVEGF therapy in eyes with diabetic macular edema and retinal vein occlusion. Invest Ophthalmol Vis Sci 58(1): 30-34.

12. Hwang TS, Gao SS, Liu L, Lauer AK, Bailey ST, et al. (2016) Automated quantification of capillary nonperfusion using optical coherence tomography angiography in diabetic retinopathy. JAMA Ophthalmol 134(4): 367-373.
13. Lin AD, Lee AY, Zhang Q, Rezaei KA, Kinyoun J, et al. (2017) Association between OCT-based microangiography perfusion indices and diabetic retinopathy severity. Br J Ophthalmol 101(7): 960-964.

14. Bhanushali D, Anegondi N, Gadde SG, Srinivasan P, Chidambara L, et al. (2016) Linking retinal microvasculature features with severity of diabetic retinopathy using optical coherence tomography angiography. Invest Ophthalmol Vis Sci 57(9): OCT519-25.

15. Sawada O, Ichiyama Y, Obata S, Ito Y, Kakinoki M, et al. (2018) Comparison between wide-angle OCT angiography and ultra-widefield fluorescein angiography for detecting non-perfusion areas and retinal neovascularization in eyes with diabetic retinopathy. Graefes Arch Clin Exp Ophthalmol 256(7): 1275-1280.

16. Hirano T, Kitahara J, Toriyama Y, Kasamatsu H, Murata T, et al. (2018) Quantifying vascular density and morphology using different sweptsource optical coherence tomography angiographic scan patterns in diabetic retinopathy. Br J Ophthalmol pii: bjophthalmol-2018-311942.

\section{Your next submission with Juniper Publishers} will reach you the below assets

- Quality Editorial service

- Swift Peer Review

- Reprints availability

- E-prints Service

- Manuscript Podcast for convenient understanding

- Global attainment for your research

- Manuscript accessibility in different formats

( Pdf, E-pub, Full Text, Audio)

- Unceasing customer service

Track the below URL for one-step submission https://juniperpublishers.com/online-submission.php 